\title{
Malignant Melanoma with Probable Smooth Muscle Differentiation
}

\author{
Aya Morimoto $^{\mathrm{a}} \quad$ Jun Asai ${ }^{\mathrm{a}} \quad$ Yusuke Wakabayashi $^{\mathrm{a}}$ Satoshi Komori \\ Keiji Hanada ${ }^{a}$ Hideya Takenaka $^{a}$ Eiichi Konishi ${ }^{b}$ Norito Katoh $^{a}$ \\ Departments of a Dermatology and ${ }^{b}$ Surgical Pathology, Graduate School of Medical \\ Science, Kyoto Prefectural University of Medicine, Kyoto, Japan
}

\section{Key Words}

Malignant melanoma $\cdot$ Smooth muscle differentiation · Divergent differentiation

\begin{abstract}
Malignant melanomas occasionally exhibit various divergent differentiation types. Of these, smooth muscle differentiation is extremely rare; only 1 case has been reported in the literature until recently. We report an extremely rare case of malignant melanoma with smooth muscle differentiation, which appeared as an amelanotic reddish nodule on the left toe.

(c) 2014 S. Karger AG, Basel
\end{abstract}

\section{Introduction}

Malignant melanomas occasionally exhibit various divergent differentiation types such as fibroblastic/myofibroblastic, schwannian and perineurial, smooth muscle, rhabdomyosarcomatous, osteocartilaginous, ganglionic and ganglioneuroblastic, neuroendocrine, and probable epithelial types $[1,2]$. Of these, smooth muscle differentiation is extremely rare; only 1 case has been reported in the literature until recently [3]. We herein report an extremely rare case of malignant melanoma with smooth muscle differentiation, which appeared as an amelanotic reddish nodule on the left toe.

\section{Case Presentation}

A 63-year-old woman presented with an ulcerated reddish nodule on the left toe (fig. 1a). No pigmentation was observed in the lesion. The patient had no other pigmented lesion, 
which was suspected as malignant melanoma. Leiomyosarcoma was highly suspected based on an incisional biopsy performed at another hospital; thus, the patient was referred to our institution. Subsequently, the left toe was amputated. Histopathologically, the neoplasm occupied the entire dermis (fig. 1b) and was composed of pleomorphic spindle cells with eosinophilic cytoplasm, blunt-ended nuclei, and perinuclear vacuoles (fig. 1c). Mitosis was frequently observed (47 mitosis/10 high-power fields). Alfa smooth muscle actin ( $\alpha$-SMA; fig. 1d) and desmin (fig. 1e) were diffusely positive. S-100 was focally positive (fig. 1f). At that time, these findings suggested a diagnosis of cutaneous leiomyosarcoma. Seven months after the amputation, the patient presented with left groin lymph node swelling. Analysis of the excised lymph node showed replacement of lymph node tissue with a cytologically uniform eosinophilic spindle cell neoplasm (fig. 1g). Immunohistochemically, the spindle cells were diffusely positive for S-100 (fig. 1h) and $\alpha$-SMA (fig. 1i). Staining for microphthalmia transcription factor (MiTF; fig. 1j) and HMB45 (fig. 1k) was focally positive. Melan-Apositive cells were scarcely observed (fig. 11). Since whole tissues were embedded in paraffin block, electron microscopic analysis could not be performed. Finally, we made the diagnosis of spindle cell melanoma with probable smooth muscle differentiation. Two years after lymph node dissection followed by 5 courses of chemotherapy (dacarbazine, nimustine hydrochloride, vincristine sulfate, and interferon beta), no local recurrence or distant metastasis was observed.

\section{Discussion}

Malignant melanomas occasionally exhibit divergent differentiation, thus resulting in difficulties in diagnosis. In this case, smooth muscle differentiation was detected by immunostaining for $\alpha$-SMA and desmin. Desmin is the intermediate filament protein associated with both smooth and skeletal muscle differentiation; therefore, desmin is the most sensitive marker for this type of differentiation. Malignant melanomas rarely show positivity for desmin, which generally represents rhabdomyoblastic differentiation [1, 4]. In contrast, smooth muscle differentiation of a melanoma can be identified by $\alpha$-SMA positivity in addition to desmin positivity [3]. In the present case, immunohistological examination of the primary lesion showed negative staining for melanoma-specific markers such as HMB45 and melan-A. Thus, we misdiagnosed the lesion as a cutaneous leiomyosarcoma until immunohistochemical analysis of the metastatic lesion showed positive staining for MiTF and HMB45. Since cutaneous leiomyosarcomas clinically behave in benign fashion in general [5], careful examination is essential to distinguish them from malignant melanomas.

In conclusion, we report a rare, but highly instructive case of malignant melanoma with smooth probable muscle differentiation. Malignant melanoma should always be considered as a differential diagnosis for such lesions, since various divergent differentiation types may be observed in melanomas.

\section{Disclosure Statement}

The authors report that they have no conflicts of interest. 
Morimoto et al.: Malignant Melanoma with Probable Smooth Muscle Differentiation

\section{References}

1 Banerjee SS, Eyden B: Divergent differentiation in malignant melanomas: a review. Histopathology 2008;52:119-129.

-2 Banerjee SS, Harris M: Morphological and immunophenotypic variations in malignant melanoma. Histopathology 2000;36:387-402.

-3 Banerjee SS, Bishop PW, Nicholson CM, Eyden BP: Malignant melanoma showing smooth muscle differentiation. J Clin Pathol 1996;49:950-951.

4 Gharpuray-Pandit D, Coyne J, Eyden B, Banerjee SS: Rhabdomyoblastic differentiation in malignant melanoma in adults: report of 2 cases. Int J Surg Pathol 2007;15:20-25.

$\rightarrow 5$ Jensen ML, Jensen OM, Michalski W, Nielsen OS, Keller J: Intradermal and subcutaneous leiomyosarcoma: a clinicopathological and immunohistochemical study of 41 cases. J Cutan Pathol 1996;23:458-463.

A.M. and J.A. contributed equally to this work. 


\section{Case Reports in Dermatology}

\begin{tabular}{l|l}
\hline Case Rep Dermatol 2014;6:16-19 \\
\hline DOI: 10.1159/000358375 & $\begin{array}{l}\text { C 2014 S. Karger AG, Basel } \\
\text { www.karger.com/cde }\end{array}$ \\
\hline
\end{tabular}

Morimoto et al.: Malignant Melanoma with Probable Smooth Muscle Differentiation
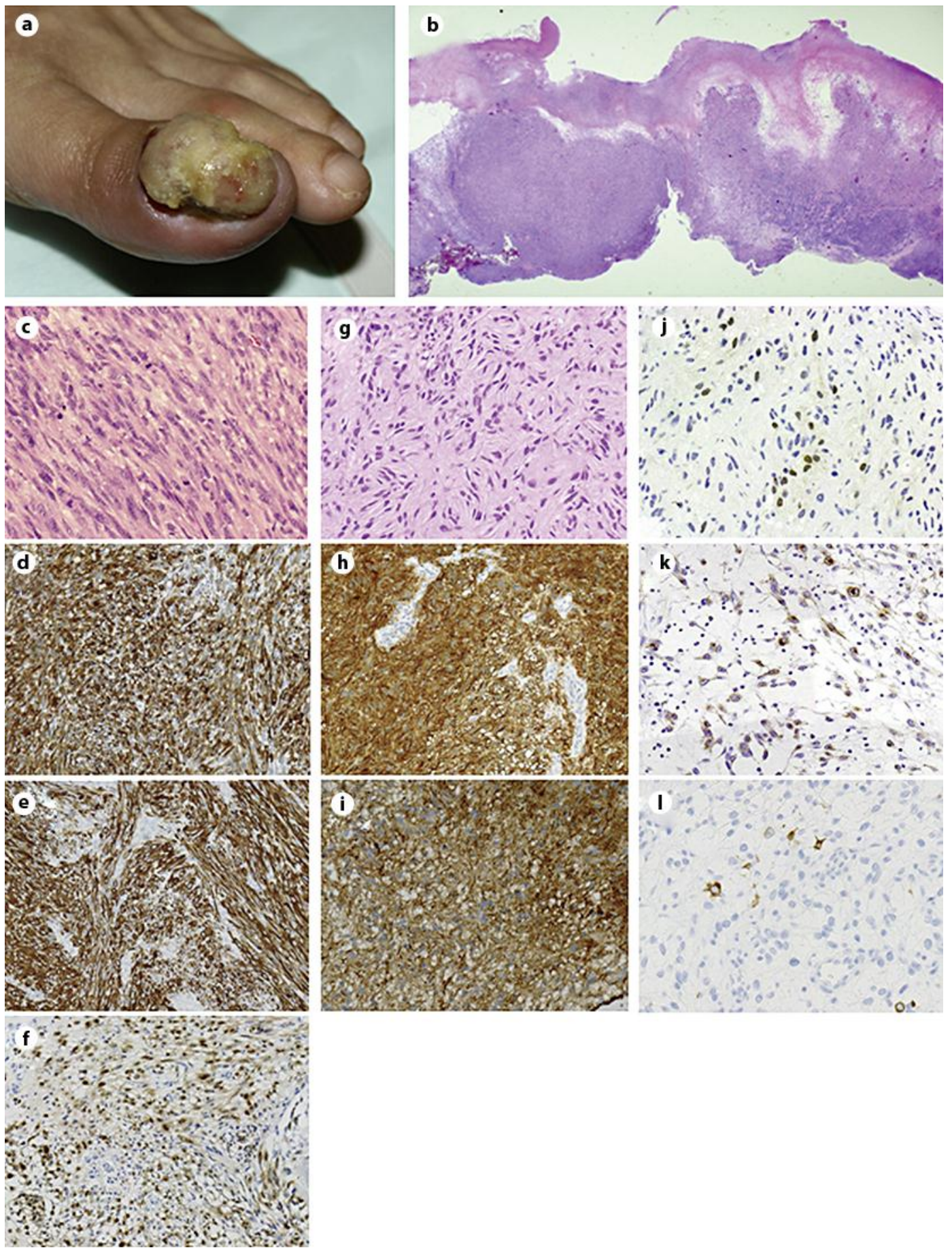

Fig. 1. a An ulcerated reddish nodule on the left toe. b The neoplasm occupied the entire dermis (hematoxylin-eosin stain). c-f Histopathological findings of the neoplasm in the left toe. Pleomorphic spindle cells with eosinophilic cytoplasm, blunt-ended nuclei, and perinuclear vacuoles (c hematoxylineosin stain). Cells were diffusely positive for $\alpha$-SMA (d) and desmin (e). Cells were focally positive for S100 (f). g-I Histopathological findings of the lymph node. g Cytologically uniform eosinophilic spindle cell neoplasm showed remarkable neural-like cytomorphology (hematoxylin-eosin staining). Cells were diffusely positive for S-100 (h) and $\alpha$-SMA (i). Cells were focally positive for MiTF (j) and HMB45 (k). Only a small number of cells were positive for melan-A (I) [original magnifications: $\times 12.5$ (b); $\times 200(\mathrm{~d}-\mathrm{i}) ; \times 400$ $(c, j-I)]$. 